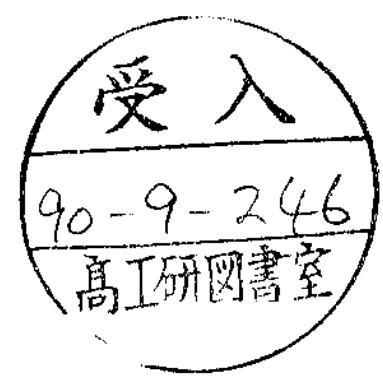

CERN-TH.5808/90

ITP-SB-90-54

\title{
Strong CP Violation \\ and \\ the Neutron Electric Dipole Moment Revisited
}

\author{
Sinya AOKI ${ }^{1}$ \\ Institute for Theoretical Physics, \\ State University of New York at Stony Brook, \\ Stony Brook, NY 11794, USA \\ and \\ Tetsuo HATSUDA ${ }^{2}$ \\ CERN, CH-1211 Geneva 23, Switzerland.
}

\begin{abstract}
The relation between the $\mathrm{CP}$ violating operator $\theta F \tilde{F}$ and its effective version $L_{C P}^{e f f}$ is reconsidered on the basis of the anomalous Ward-Takahashi (WT) identity. The consistency of the previous phenomenological calculations of the neutron electric dipole moment (NEDM) with the WT identity is critically examined, and a new consistent evaluation of NEDM is given. We found NEDM at the tree level of the hadron fields, which is comparable in magnitude with the result of the one-loop calculation.

\footnotetext{
${ }^{1}$ AOKI@SUNYSBNP.BITNET

${ }^{2}$ THATSUDA@CERNVM.BITNET. On leave of absence from Department of Physics,
} State University of New York at Stony Brook, Stony Brook, NY 11794, USA. Address after September 1, 1990; Institute for Nuclear Theory, University of Washington, Seattle 98185, USA.
\end{abstract}



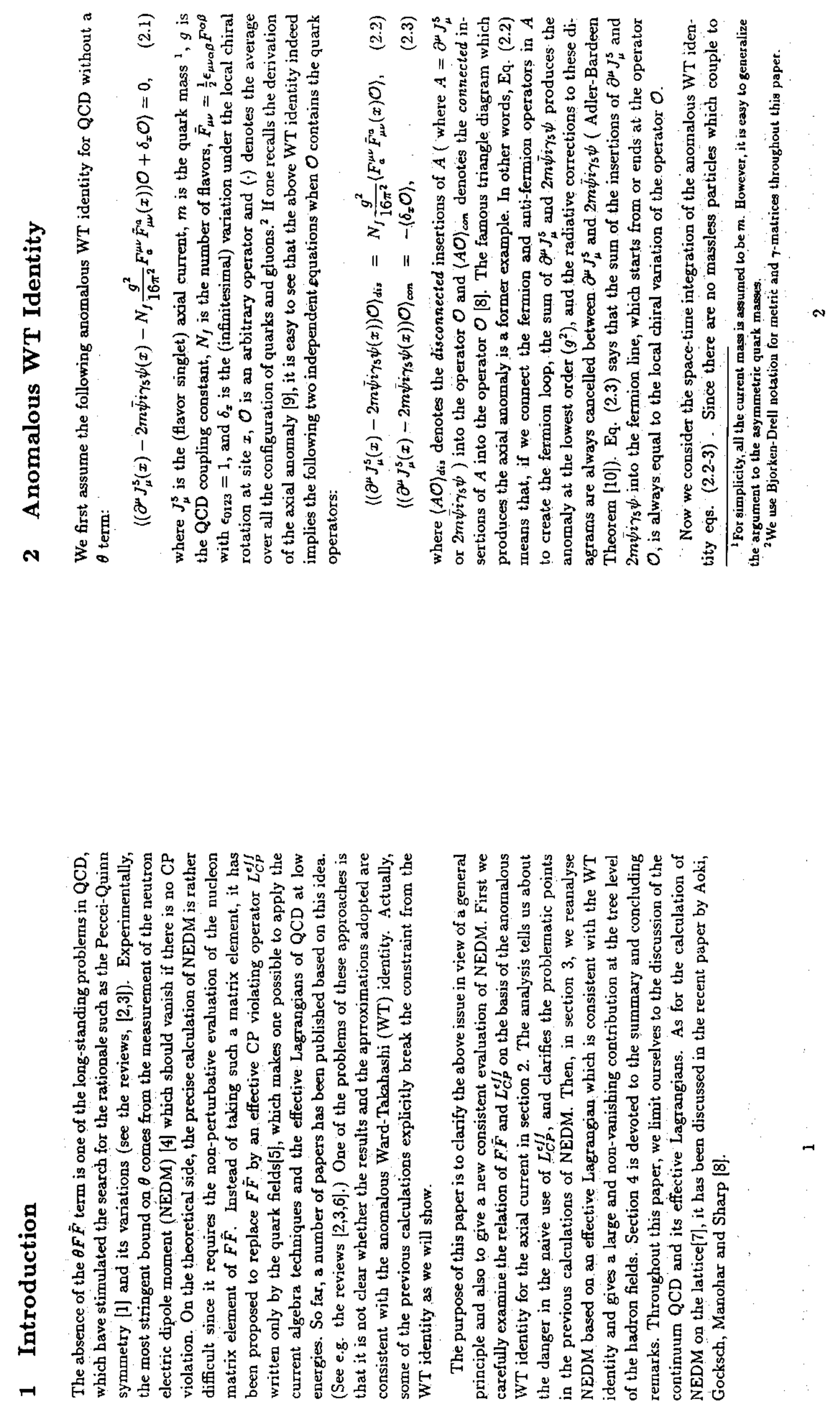

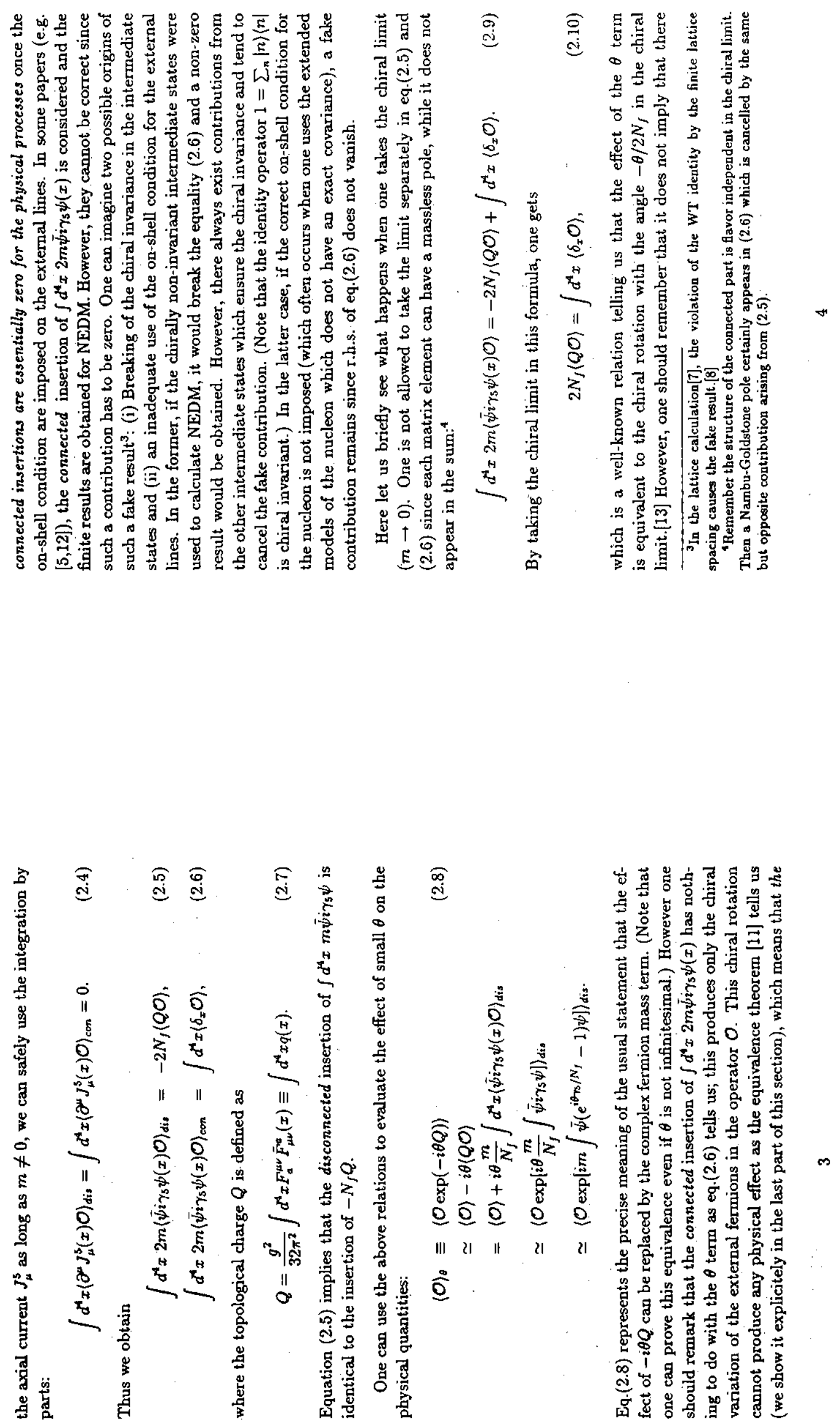

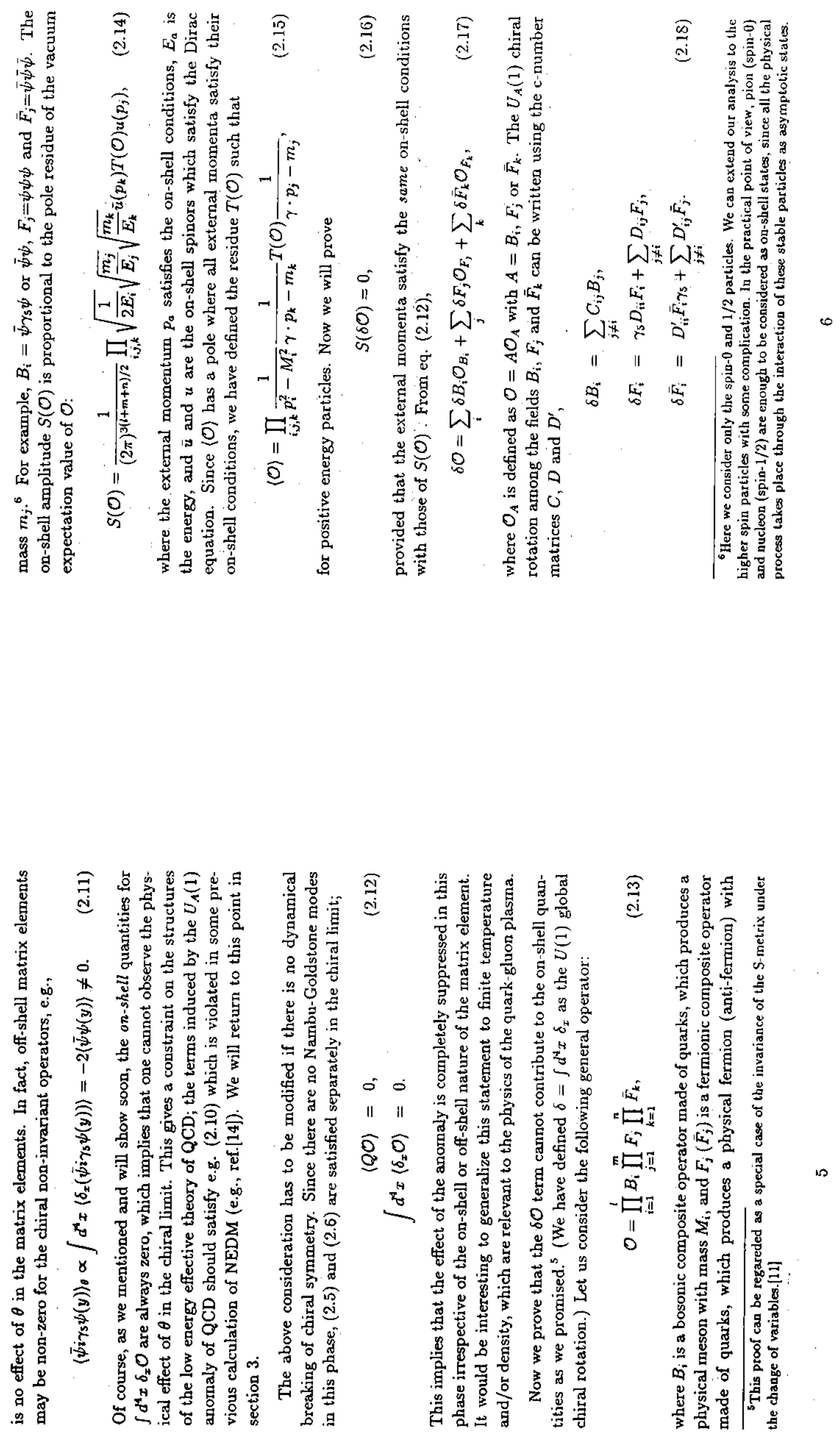

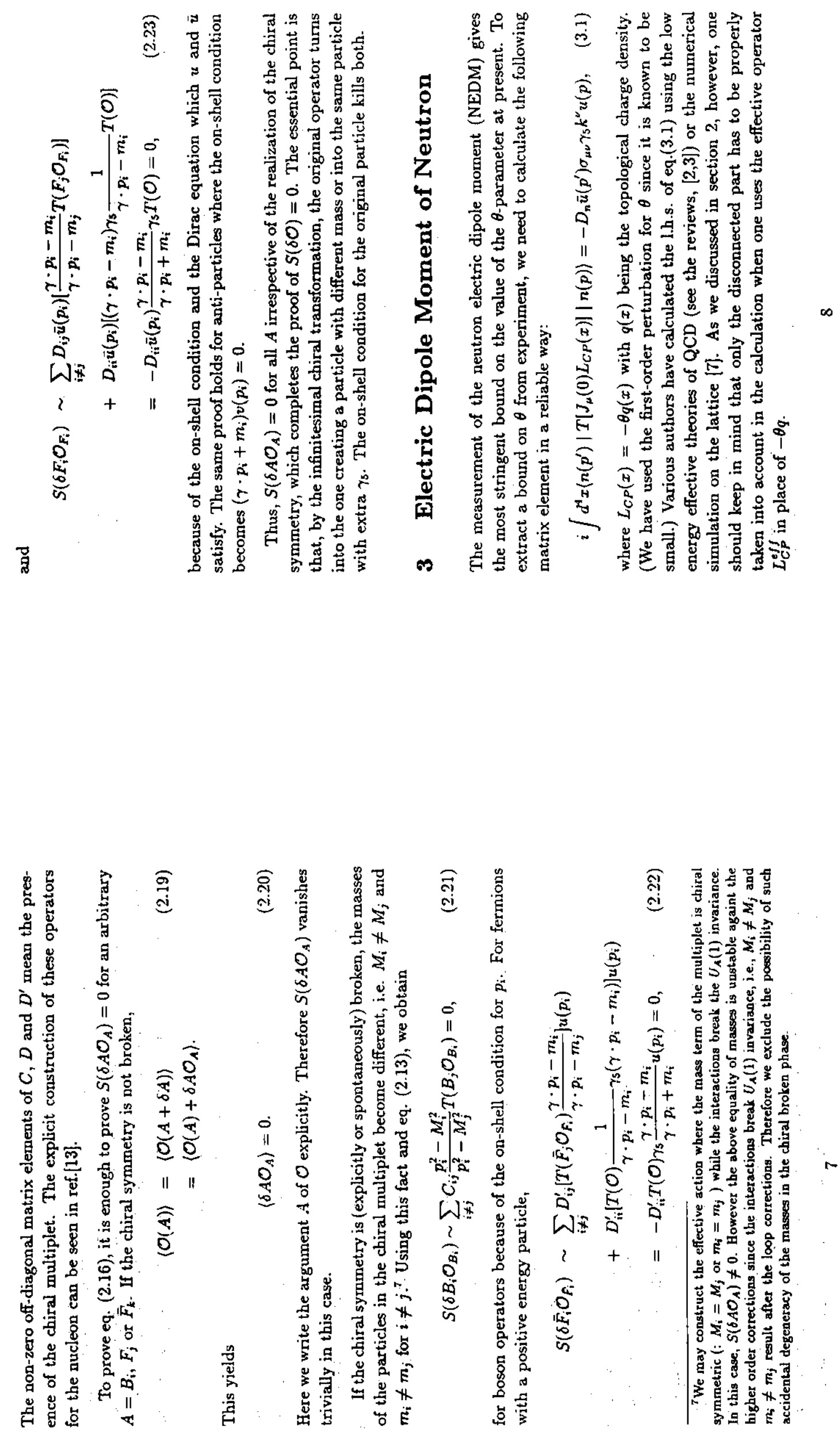

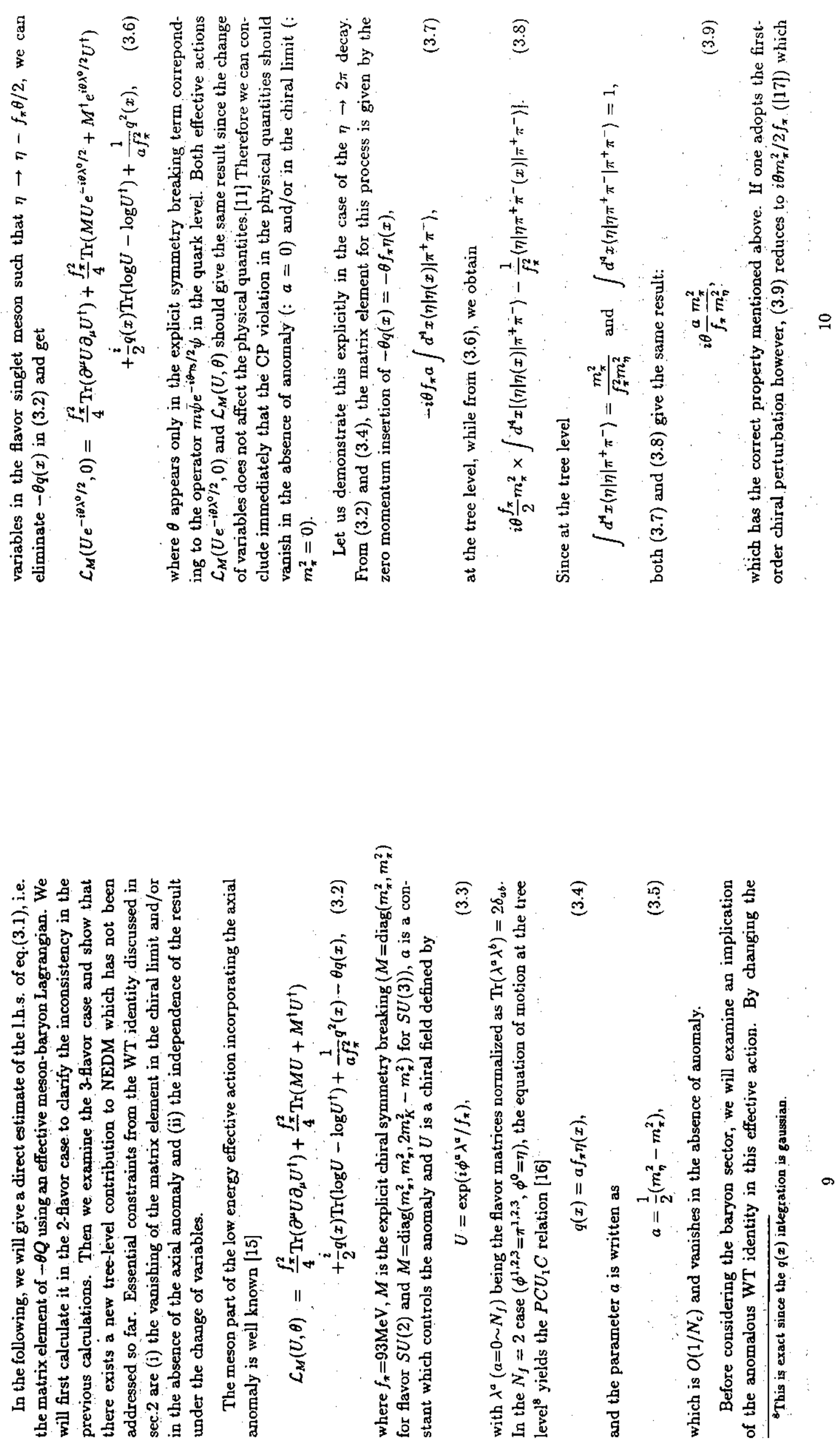

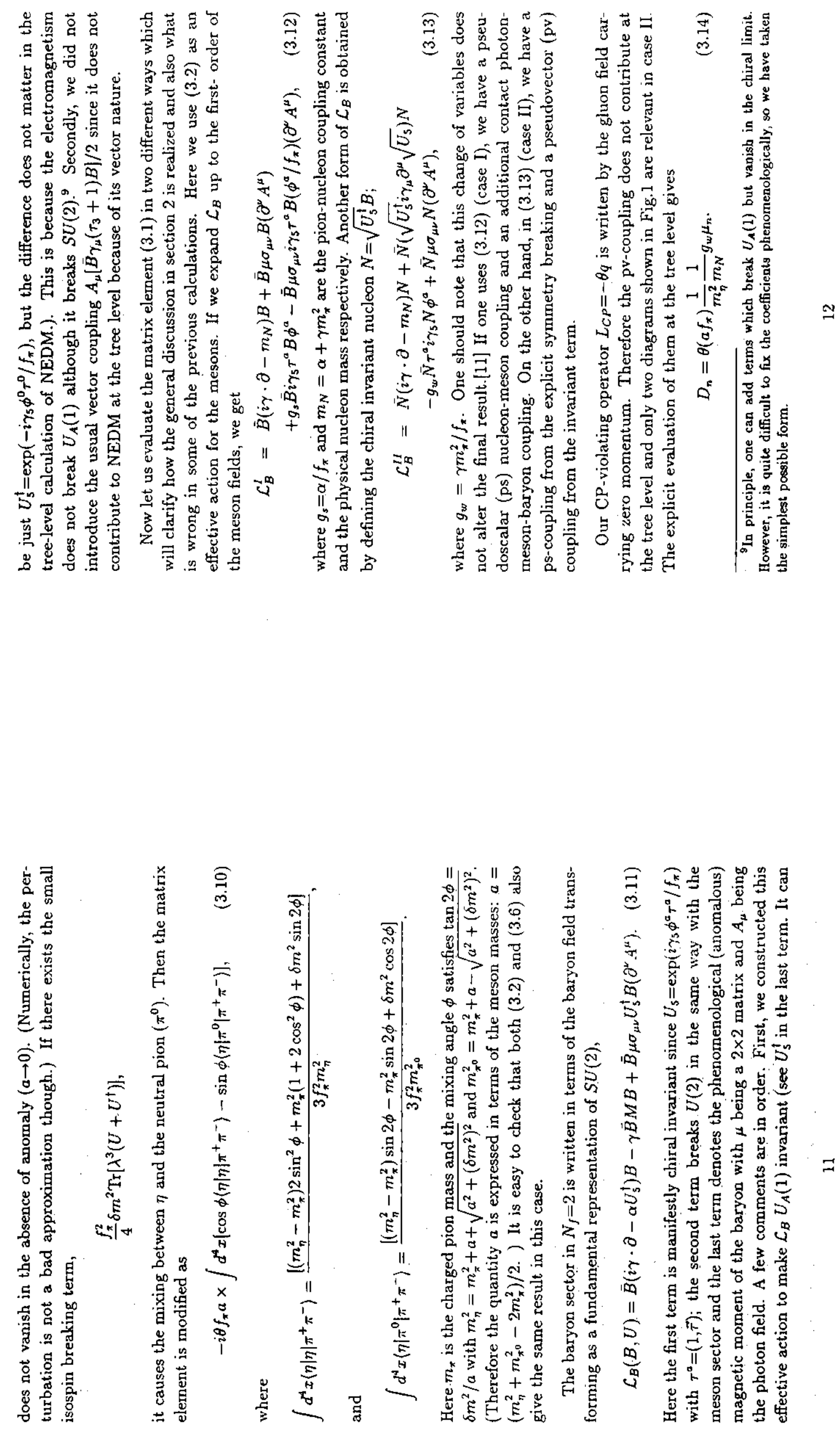

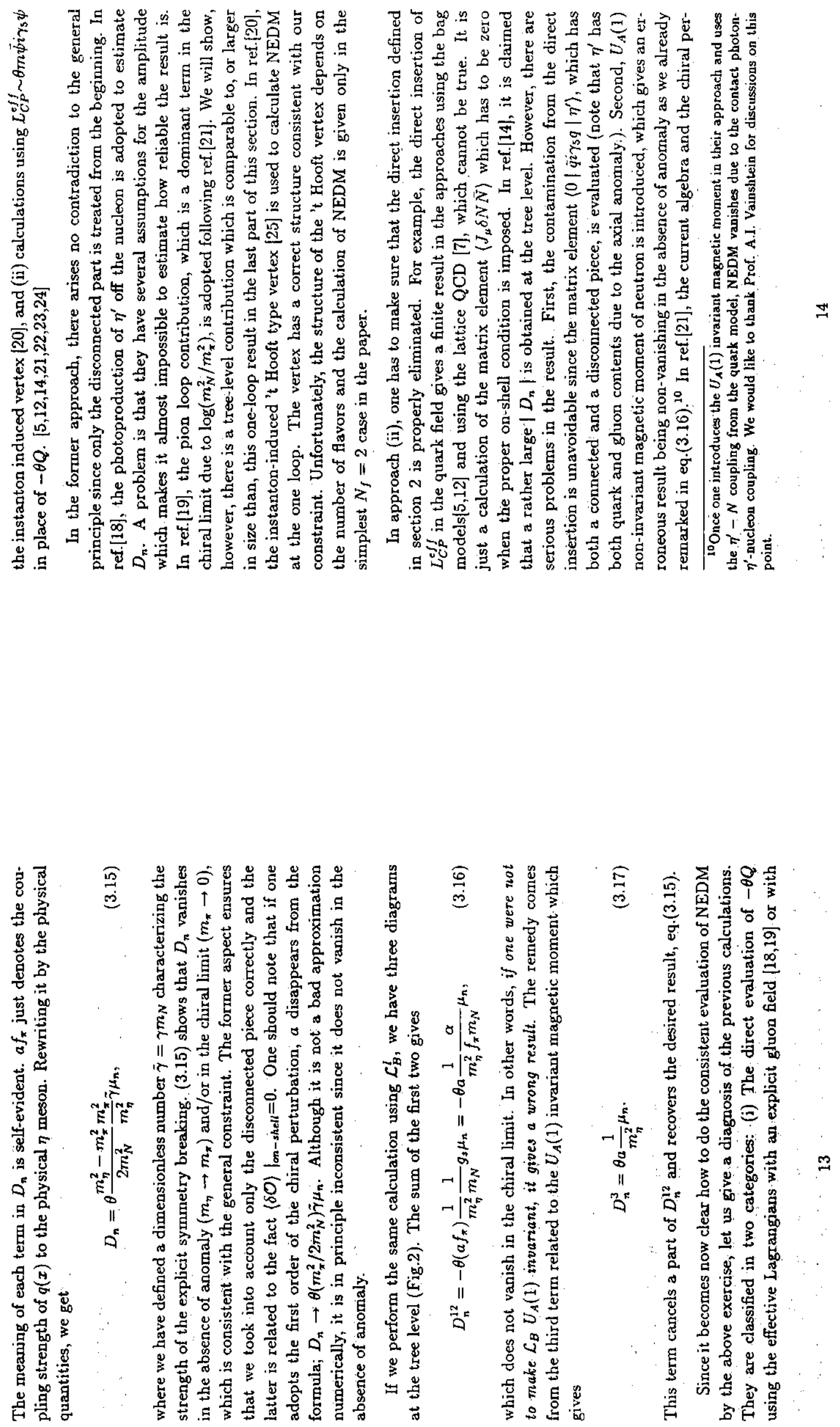

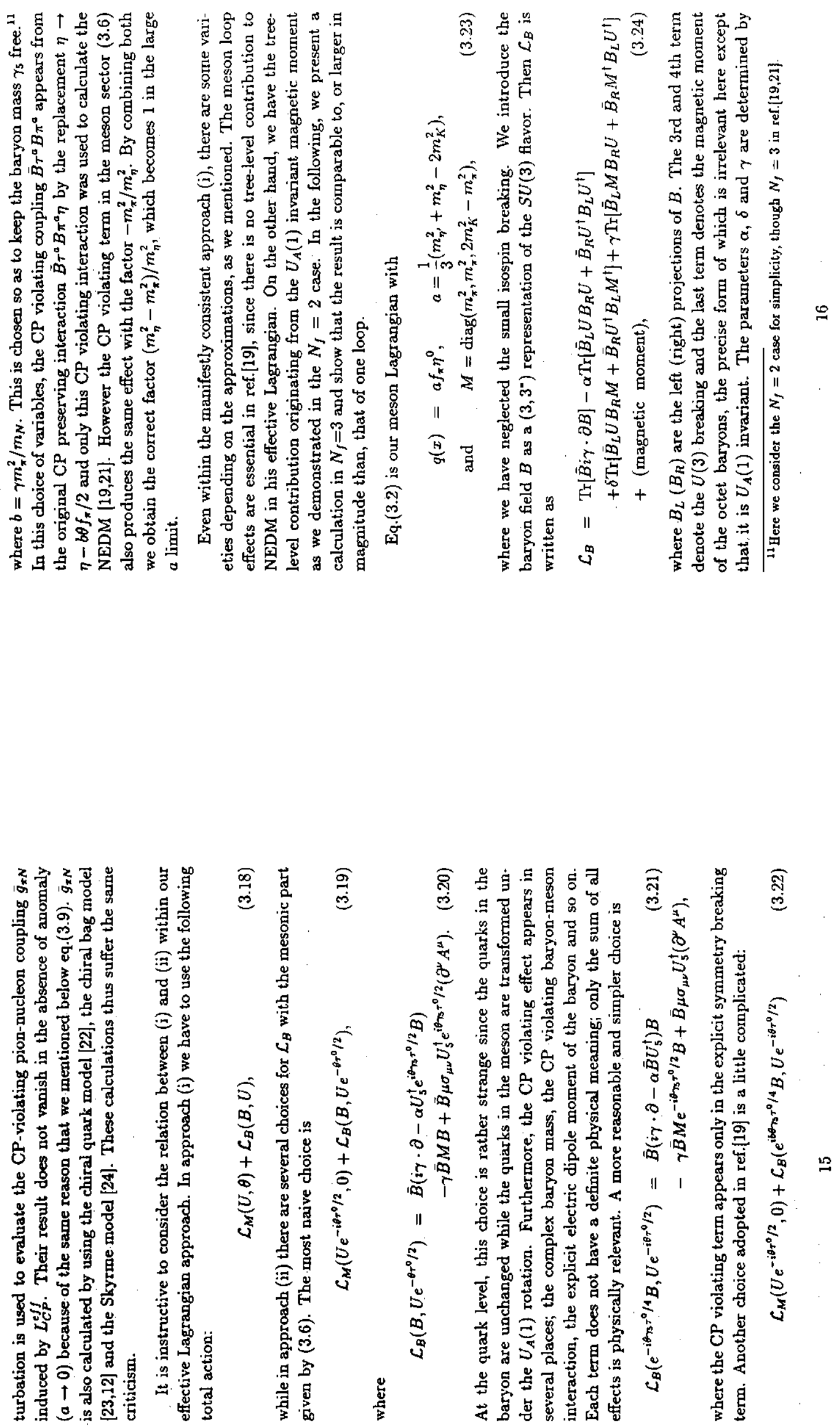

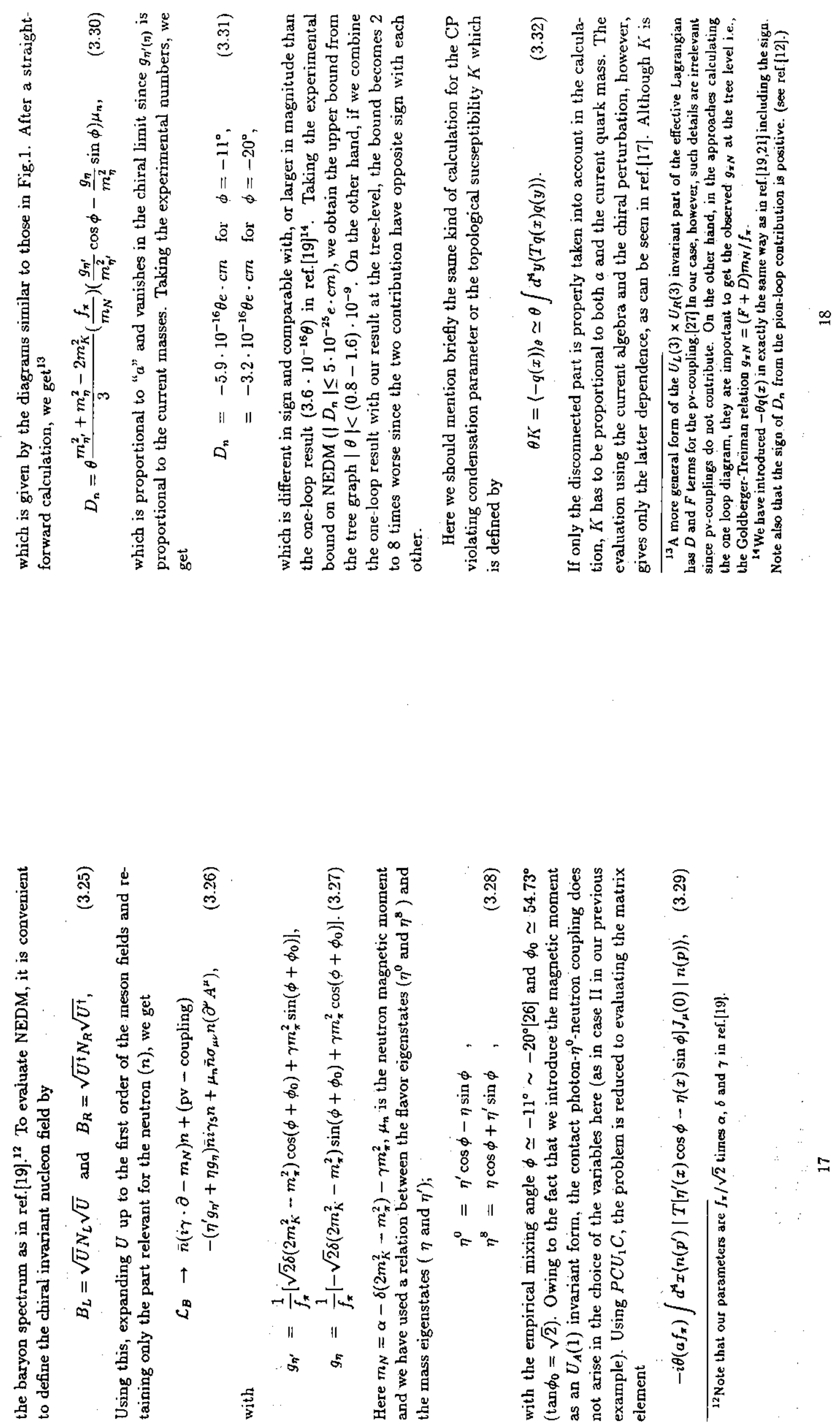

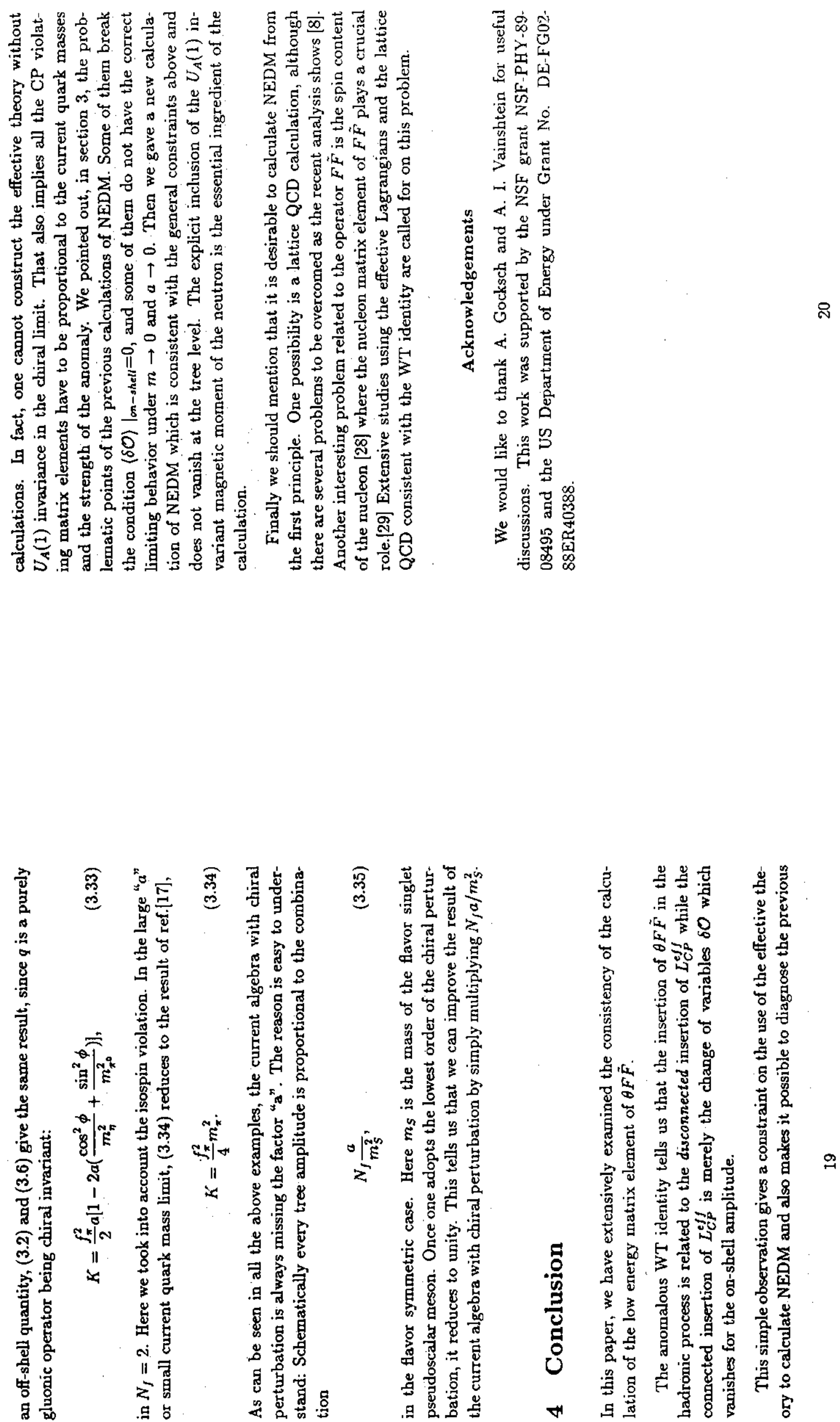

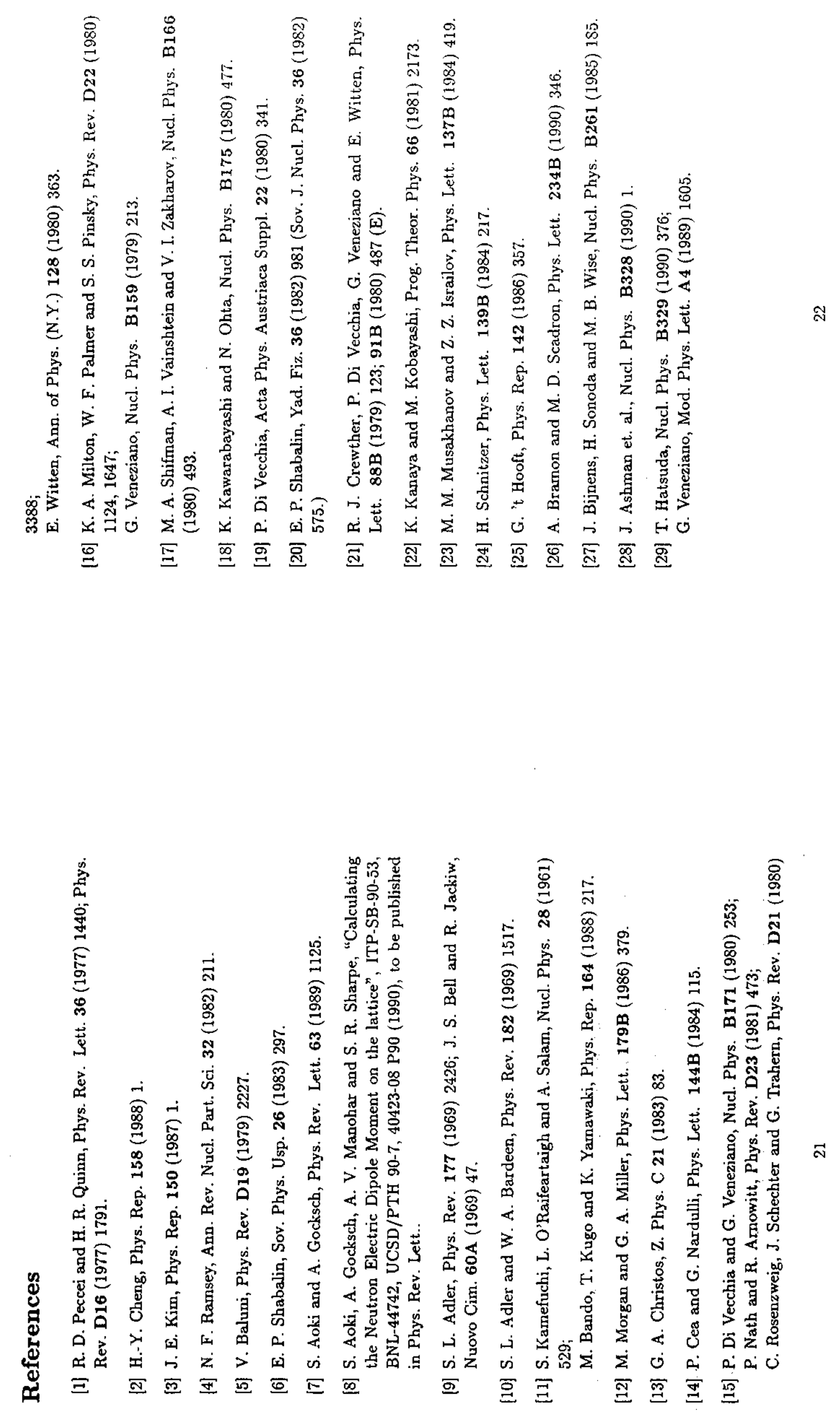


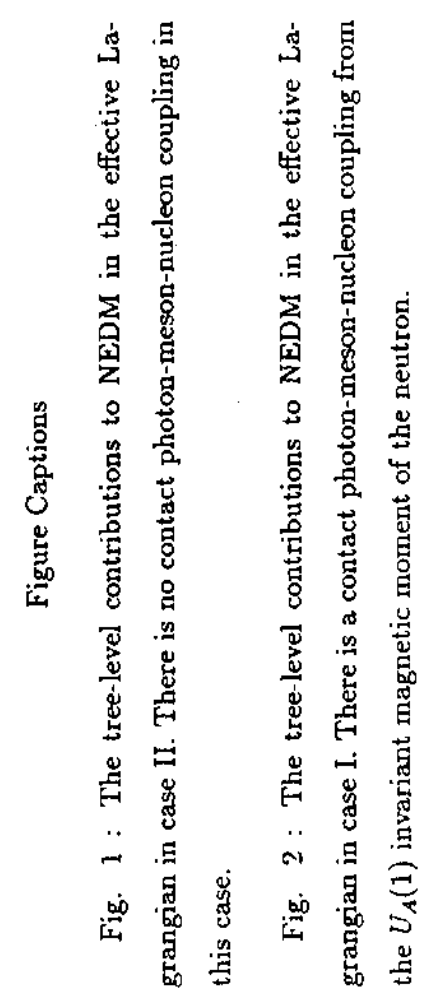


